\title{
Szymon Frankowski
}

\section{BICONSEQUENCES}

\begin{abstract}
. $\quad p$-consequence (plausible consequence; see [2]) allows for a formulation of non-deductive reasonings, i.e., such where the conclusion has weaker justification then assumptions and thus when added to the set of assumptions results in its extension. But theoretical modesty of $p$-consequence operation does not tell the difference between "good" and "worse" conclusions. Therefore the bisconsequence is introduced.
\end{abstract}

Keywords: Bisconsequence, $p$-consequence, bimatrix, $p$-matrix, biinference, biproof.

\section{Preliminaries}

Deductive inference in the sense of Ajdukiewicz [1] requires that a conclusion has degree of sureness at least the same as the degree of the weakest among the premisses. The sentence ' $a+b=0$ ' is true in the same way as the sentences ' $a=c$ ' and ' $b=-c$ ' (in the standard meaning of the symbols ' + ' and ' - '). This point of view is expressed by the third Tarskian condition of consequence operation (i.e. idempotency). In the opposite, in plausible inference, the degree of sureness of conclusion can be smaller than the degree of assumption. In the classic example the sentence 'It was raining' can be treated as having worse justification than 'The streets are wet'. Moreover, the sentence 'My garden is wet' is quite a possible conclusion from the sentence 'It was raining', but it should be considered as much worse conclusion from the assumption 'Streets are wet' (if the observer is not seeing his own garden at the moment of making the statement). So, plausible inference does not fulfil the idempotency condition. Formal characterization of plausible (non-deductive) 
inference contained in [2] requires that such an inference must have two relevant properties (for any sets of formulas $X$ and $Y$ of a fixed propositional language): $X \subseteq Z(X)$ and if $X \subseteq Y$, then $Z(X) \subseteq Z(Y)$ (it is the so-called $p$-consequence operation).

This work can be treated as a attempt of generalization of $p$-consequence operation. For any $p$-consequence there is a problem to distinguish better and worse justified sentences. For example, given a formula $\alpha \in Z(X)$ we do not know whether $\alpha$ is well justified as possible or not. This lack can be supplemented by considering an operation on a disjoint sum of the same language, i.e. $L \oplus L$. When we dispose of the set $X \oplus Y$ then the first component contains assumptions having the best degree of sureness, and the second one - not-rejected formulas. Similarly, when "consequence" of $X \oplus Y$ is of the form $N(X \oplus Y)=U \oplus V$, then the same remarks concern the sets $U$ and $V$.

In this paper we are using the following notation. For any sets $X_{1}$ and $X_{2}$ by $X_{1} \oplus X_{2}$ we mean their disjoint sum, i.e.:

$$
X_{1} \oplus X_{2}:=\left\{\langle x, 1\rangle: x \in X_{1}\right\} \cup\left\{\langle x, 2\rangle: x \in X_{2}\right\} .
$$

For $t_{0} \in\{1,2\}$, by $i_{t_{0}}: X_{t_{0}} \hookrightarrow X_{1} \oplus X_{2}$, s.t. $i_{t_{0}}(x)=\left\langle x, t_{0}\right\rangle$, we mean inclusion into $t_{0}$-component of the disjoint sum $X_{1} \oplus X_{2}$. Notice that $\emptyset=\emptyset \oplus \emptyset, X_{1} \oplus \emptyset=\left\{\langle x, 1\rangle: x \in X_{1}\right\}$ and $\emptyset \oplus X_{2}=\left\{\langle x, 2\rangle: x \in X_{2}\right\}$. Moreover, every subset of $X_{1} \oplus X_{2}$ is a disjoint union of some subsets of $X_{1}$ and $X_{2}$. Indeed, if $\boldsymbol{X} \in \mathcal{P}\left(X_{1} \oplus X_{2}\right)$, then for $t_{0} \in\{1,2\}$ we put $\boldsymbol{X}_{\left(t_{0}\right)}:=i_{t_{0}}^{-1}(\boldsymbol{X})$, i.e.:

$$
\boldsymbol{X}_{\left(t_{0}\right)}:=\left\{x:\left\langle x, t_{0}\right\rangle \in \boldsymbol{X}\right\} .
$$

Of course $\boldsymbol{X}_{\left(t_{0}\right)} \subseteq X_{t_{0}}$ and $\boldsymbol{X}=\boldsymbol{X}_{(1)} \oplus \boldsymbol{X}_{(2)}$.

For variables of disjoint unions of sets we will use bold letters: $\boldsymbol{X}$, $\boldsymbol{Y}, \boldsymbol{Z}$. It is obvious that:

Lemma 1.1. For all sets $X_{1}$ and $X_{2}: X_{1}=\left(X_{1} \oplus X_{2}\right)_{(1)}$ and $X_{2}=$ $\left(X_{1} \oplus X_{2}\right)_{(2)}$. Hence, for any disjoint union $\boldsymbol{X}$ :

(i) if $\boldsymbol{X}=X_{1} \oplus X_{2}$, then $\boldsymbol{X}_{(1)}=X_{1}$ and $\boldsymbol{X}_{(2)}=X_{2}$;

(ii) $\boldsymbol{X}=\boldsymbol{X}_{(1)} \oplus \boldsymbol{X}_{(2)}$.

For any pair of mappings $f_{1}: X_{1} \longrightarrow Y$ and $f_{2}: X_{2} \longrightarrow Y$, we define a new mapping $f_{1} \uplus f_{2}: X_{1} \oplus X_{2} \longrightarrow Y$, by putting for any $\langle x, i\rangle \in X_{1} \oplus X_{2}$ :

$$
\left(f_{1} \uplus f_{2}\right)(\langle x, i\rangle):=f_{i}(x) .
$$


Moreover, for any pair of mappings $f_{1}: X_{1} \longrightarrow Y_{1}$ and $f_{2}: X_{2} \longrightarrow Y_{2}$ we define a new mapping $\left(f_{1} \oplus f_{2}\right): X_{1} \oplus X_{2} \longrightarrow Y_{1} \oplus Y_{2}$, by putting for any $\langle x, i\rangle \in X_{1} \oplus X_{2}$ :

$$
\left(f_{1} \oplus f_{2}\right)(x, i):=\left\langle f_{i}(x), i\right\rangle
$$

\section{Biconsequence}

Definition 2.1. Assume that $\mathfrak{L}=\left\langle L, f_{1}, \ldots, f_{n}\right\rangle$ is a propositional language generated by the set of atoms At. Then by biconsequence for $\mathfrak{L}$ we understand any mapping $N: \mathcal{P}(L \oplus L) \longrightarrow \mathcal{P}(L \oplus L)$ such that for any disjoint unions $\boldsymbol{X}, \boldsymbol{Y}$ from $\mathcal{P}(L \oplus L)$, the following conditions hold:

(i) $\boldsymbol{X} \subseteq N(\boldsymbol{X})$,

(ii) $N(\boldsymbol{X}) \subseteq N(\boldsymbol{Y})$, whenever $\boldsymbol{X} \subseteq \boldsymbol{Y}$,

(iii) $N\left(N(\boldsymbol{X})_{(1)} \oplus \boldsymbol{X}_{(2)}\right) \subseteq N(\boldsymbol{X})$.

Brief analysis of the above definition allows to spell out non-formal intentions. In each disjoint sum sets of formulas $X_{1} \oplus X_{2}$ we indicate assumptions from the first, the best founded (see similarities between (iii) and the condition $C(C(X)) \subseteq C(X)$ in the classical theory of consequence), and from the second - worse but not the worst.

Moreover, if for every substitution $e$ of the language $\mathfrak{L}$ and every $\boldsymbol{X} \in \mathcal{P}(L \oplus L)$ we have that:

$$
(e \oplus e)(N(\boldsymbol{X})) \subseteq N\left(e\left(\boldsymbol{X}_{(1)}\right) \oplus e\left(\boldsymbol{X}_{(2)}\right)\right),
$$

then $N$ will be called structural.

For now we do not demand stronger condition than this: for all substitutions $e_{1}, e_{2},\left(e_{1} \oplus e_{2}\right)(N(\boldsymbol{X})) \subseteq N\left(e_{1}\left(\boldsymbol{X}_{(1)}\right) \oplus e_{2}\left(\boldsymbol{X}_{(2)}\right)\right)$, because it seems to be factitious in the linguistic practice. We assume that a plausible reasoning is expressible in the same language as deductive - with every consequence of that fact - when we substitute some formulas for propositional variables to say something about reality. We distinguish only the degrees of certainty.

As in the standard theory of consequence, $N$ is finitary iff $N(\boldsymbol{X})=$ $\bigcup\{N(\boldsymbol{Y}): \boldsymbol{Y} \in \operatorname{Fin}(\boldsymbol{X})\}$, where $\operatorname{Fin}(\boldsymbol{X})$ stands for the family of finite subsets of $\boldsymbol{X}$ (including the empty set).

Theory of $p$-consequence (see e.g. [2]) contains the notion of $p$-matrix, that is defined to be a structure $\mathfrak{M}=\left\langle M, F_{1}, \ldots, F_{n}, D_{1}, D_{*}\right\rangle$, where 
$\left\langle M, F_{1}, \ldots, F_{n}\right\rangle$ is an algebra of interpretations similar to a given language $\mathfrak{L}=\left\langle L, f_{1}, \ldots, f_{n}\right\rangle, D_{1}$ and $D_{*}$, with $D_{1} \subseteq D_{*} \subseteq M$, are the sets of distinguished values of two kinds:

- for $D_{1}$ : values corresponding to the maximal possible sureness,

- for $D_{*}$ : values of the smaller degree of sureness.

Moreover, $p$-consequence $Z_{\mathfrak{M}}$ is defined by a $p$-matrix $\mathfrak{M}$ in the following manner:

$\alpha \in Z_{\mathfrak{M}}(X) \quad$ iff $\quad h(X) \subseteq D_{1}$ implies $h \alpha \in D_{*}$, for any homomorphisms $h: \mathfrak{L} \longrightarrow \mathfrak{M}$.

How to interpret the above definition? Its explanation is simple and rather intuitive: $\alpha$ is a $p$-conclusion of the set $X$ iff every interpretation that sends all the premisses into the smaller set $D_{1}$ (i.e. such one which values $X$ in the best possible manner) does not take out $\alpha$ outside $D_{*}$ (that is $\alpha$ is not rejected).

By a bimatrix for the language $\mathfrak{L}$ we shall understand a structure $\mathfrak{M}=\left\langle M, F_{1}, \ldots, F_{n}, E, D_{1}, D_{*}\right\rangle$, where $\left\langle M, F_{1}, \ldots, F_{n}\right\rangle$ is algebra similar to $\mathfrak{L}, E \subseteq M$ and $D_{1} \subseteq D_{*} \subseteq M$.

Every bimatrix $\mathfrak{M}=\left\langle M, F_{1}, \ldots, F_{n}, E, D_{1}, D_{*}\right\rangle$ determines the operation of biconsequence $N_{\mathfrak{M}}: \mathcal{P}(L \oplus L) \longrightarrow \mathcal{P}(L \oplus L)$ in the following way. For any $\boldsymbol{X} \in \mathcal{P}(L \oplus L)$ (see Lemma 1.1):

$$
N_{\mathfrak{M}}(\boldsymbol{X}):=N_{\mathfrak{M}}(\boldsymbol{X})_{(1)} \oplus N_{\mathfrak{M}}(\boldsymbol{X})_{(2)},
$$

where for any $\alpha \in L$ :

$$
\begin{aligned}
& \alpha \in N_{\mathfrak{M}}(\boldsymbol{X})_{(1)} \text { iff } \\
& \quad \forall_{h \in \operatorname{Hom}(\mathfrak{L}, \mathfrak{M})}\left(h\left(\boldsymbol{X}_{(1)}\right) \subseteq E \& h\left(\boldsymbol{X}_{(2)}\right) \subseteq D_{1} \Rightarrow h(\alpha) \in E\right),
\end{aligned}
$$

and

$$
\begin{aligned}
& \alpha \in N_{\mathfrak{M}}(\boldsymbol{X})_{(2)} \text { iff } \\
& \quad \forall_{h \in \operatorname{Hom}(\mathfrak{L}, \mathfrak{M})}\left(h\left(\boldsymbol{X}_{(1)}\right) \subseteq E \& h\left(\boldsymbol{X}_{(2)}\right) \subseteq D_{1} \Rightarrow h(\alpha) \in D_{*}\right) .
\end{aligned}
$$

Let us briefly comment the above definition. The assumptions are divided into two sets, i.e., components of disjoint sum $\boldsymbol{X}=X_{1} \oplus X_{2}$. If 
$h$ is an interpretation of the propositional language which sends $X_{1}$ into the set $E$ (of distinguished values for the left side) and sends $X_{2}$ into $D_{1}$ (the set of strongly distinguished values of the left side), then the formulas from $N_{\mathfrak{M}}(\boldsymbol{X})_{(1)}$ must be mapped into $E$. This naturally behaves as a matrix consequence operation. Similarly, the second component of the sum behaves like a matrix $p$-consequence operation. One can ask whether it should rather be forced that $D_{1}=E$. Although, it sounds quite reasonably, we have decided for a more general version complete w.r.t. Definition 2.1. (see also Theorem 2.1.)

Proposition 2.1. $N_{\mathfrak{M}}$ is structural consequence operation.

Proof. We put $N=N_{\mathfrak{M}}$. First two conditions of the definition of biconsequence are obvious. Let $\alpha \in N\left(N(\boldsymbol{X})_{(1)} \oplus \boldsymbol{X}_{(2)}\right)_{(i)}$, where $i=1,2$. Of course, $\left(N(\boldsymbol{X})_{(1)} \oplus \boldsymbol{X}_{(2)}\right)_{(1)}=N(\boldsymbol{X})_{(1)}$ and $\left(N(\boldsymbol{X})_{(1)} \oplus \boldsymbol{X}_{(2)}\right)_{(2)}=\boldsymbol{X}_{(2)}$. We also suppose that for some $h \in \operatorname{Hom}(\mathfrak{L}, \mathfrak{M})$ we have: $h\left(\boldsymbol{X}_{(1)}\right) \subseteq E$ and $h\left(\boldsymbol{X}_{(2)}\right) \subseteq D_{1}$. Then, by definition of $N(\boldsymbol{X})_{(1)}$, for any $\beta \in N(\boldsymbol{X})_{(1)}$, we have $h(\beta) \in E$. Thus, $h\left(N(\boldsymbol{X})_{(1)}\right) \subseteq E$. Since by assumption $h\left((\boldsymbol{X})_{(2)}\right) \subseteq D_{1}$, so $h(\alpha) \in E$, when $i=1$, and $h(\alpha) \in D_{*}$, when $i=2$. Hence $\alpha \in N(\boldsymbol{X})_{(i)}$. Thus, $N\left(N(\boldsymbol{X})_{(1)} \oplus \boldsymbol{X}_{(2)}\right) \subseteq N(\boldsymbol{X})$.

The proof the structurality of $N$ is straightforward.

Proposition 2.2. For every class $\boldsymbol{N}$ of biconsequences operation $\bigwedge \boldsymbol{N}$ defined by $(\bigwedge \boldsymbol{N})(\boldsymbol{X}):=\bigcap_{N \in \boldsymbol{N}} N(\boldsymbol{X})$ is a biconsequence.

Proof. For every $N_{0} \in \boldsymbol{N}$ we have $(\bigwedge \boldsymbol{N})\left[((\bigwedge \boldsymbol{N})(\boldsymbol{X}))_{(1)} \oplus(\boldsymbol{X})_{(2)}\right]=$ $\bigcap_{N \in \boldsymbol{N}} N\left[((\bigwedge \mathrm{N})(\boldsymbol{X}))_{(1)} \oplus(\boldsymbol{X})_{(2)}\right] \subseteq N_{0}\left[\left(N_{0}(\boldsymbol{X})\right)_{(1)} \oplus(\boldsymbol{X})_{(2)}\right] \subseteq N_{0}(\boldsymbol{X})$. Thus $(\bigwedge \boldsymbol{N})\left[((\bigwedge \boldsymbol{N})(\boldsymbol{X}))_{(1)} \oplus(\boldsymbol{X})_{(2)}\right] \subseteq(\bigwedge \boldsymbol{N})(\boldsymbol{X})$. As the conditions (i) and (ii) are obvious we omit them.

Corollary 2.1. For every class BM of bimatrices operation

$$
N_{B M}:=\bigwedge\left\{N_{\mathfrak{M}}: \mathfrak{M} \in B M\right\}
$$

forms a structural biconsequence.

For any biconsequence $N$ we put

$$
\mathbb{L}(N):=\left\{\left\langle L, f_{1}, \ldots, f_{n}, N(\boldsymbol{X})_{(1)}, \boldsymbol{X}_{(2)}, N(\boldsymbol{X})_{(2)}\right\rangle\right\}_{\boldsymbol{X} \in \mathcal{P}(L \oplus L)}
$$

Naturally $\mathbb{L}(N)$ is a subclass of the class of all bimatrices for $\mathfrak{L}$. 
TheOREM 2.1. $N$ is structural iff $N_{\mathbb{L}(N)}=N$.

Proof. " $\Rightarrow$ " $(\supseteq)$ Assume that $\alpha \in N(\boldsymbol{X})_{(i)}(i=1,2)$ and let $e \oplus e(\boldsymbol{X}) \subseteq$ $N(\boldsymbol{Y})_{(1)} \oplus \boldsymbol{Y}_{(2)}$. Thus, $e(\alpha) \in N(\boldsymbol{Y})_{(i)}$, since $e \oplus e N(\boldsymbol{X}) \subseteq N(e \oplus e(\boldsymbol{X})) \subseteq$ $N\left[N(\boldsymbol{Y})_{(1)} \oplus \boldsymbol{Y}_{(2)}\right]=N(\boldsymbol{Y})$ and $\alpha \in N_{\mathbb{L}(N)}(\boldsymbol{X})_{(i)}$.

$(\subseteq)$ If $\alpha \notin N(\boldsymbol{X})_{(i)}$, then $(\operatorname{id} \oplus \operatorname{id})(\boldsymbol{X}) \subseteq N(\boldsymbol{X})_{(1)} \oplus \boldsymbol{X}_{(2)}$ and $\operatorname{id}(\alpha) \notin$ $N(\boldsymbol{X})_{(i)}$. Finally, $\alpha \notin N_{\mathbb{L}(N)}(\boldsymbol{X})_{(i)}$.

" $\Leftarrow$ " By Corollary 2.1.

Every pair of the form $C \oplus Z$, where $C$ is a consequence, $Z$ is a $p$-consequence operation, and $(C \oplus Z)(\boldsymbol{X}):=C\left(\boldsymbol{X}_{(1)}\right) \oplus Z\left(\boldsymbol{X}_{(2)}\right)$ is a biconsequence. But not every biconsequence has a representation such that - it is enough to $N\left(X_{1} \oplus X_{2}\right)_{(2)} \neq N\left(Y_{1} \oplus X_{2}\right)_{(2)}$ for some $X_{1}, Y_{1}, X_{2} \in \mathcal{P}(L)$.

Definition 2.2. By biinference we understand an arbitrary member of the set $\bigcup_{k \in \mathbf{N}}(L \times\{1, *: *, *: 1\})^{k}$.

DEFINITION 2.3. $r$ is called birule iff $r$ a non-empty set of biinferences.

Definition 2.4. Biinfence $\left(a_{1}, \ldots, a_{n}\right)$ is a biproof of $\langle\alpha, x\rangle$ (where $x \in$ $\{1, *: *, *: 1\})$ based on the set $\boldsymbol{R}$ of birules from the set $\boldsymbol{X}$ iff $a_{n}=\langle\alpha, x\rangle$ and for every $i \in\{1, \ldots, n\}$ at last one of the following conditions holds:

(i) $\operatorname{pr}_{1}\left(a_{i}\right) \in \boldsymbol{X}_{(1)}$ and $\operatorname{pr}_{2}\left(a_{i}\right)=1 ;^{1}$

(ii) $\operatorname{pr}_{1}\left(a_{i}\right) \in \boldsymbol{X}_{(2)}$ and $\operatorname{pr}_{2}\left(a_{i}\right)=*: 1$;

(iii) for some $\left\{b_{1}, \ldots, b_{k}\right\} \subseteq\left\{a_{1}, \ldots, a_{i-1}\right\},\left(b_{1}, \ldots, b_{k}, a_{i}\right) \in \bigcup \boldsymbol{R}$.

For any set $\boldsymbol{R}$ of birules we define $N_{\boldsymbol{R}}: \mathcal{P}(L \oplus L) \longrightarrow \mathcal{P}(L \oplus L)$ :

$N_{\boldsymbol{R}}(\boldsymbol{X}):=\{\langle\alpha, 1\rangle$ : exists biproof of $\langle\alpha, 1\rangle$ based on $\boldsymbol{R}$ from $\boldsymbol{X}\} \cup$ $\{\langle\alpha, 2\rangle$ : exists biproof of $\langle\alpha, x\rangle$, where $x \in\{*: *, *: 1\}$,

based on $\boldsymbol{R}$ from $\boldsymbol{X}$ \}.

Theorem 2.2. For any set of birules $\boldsymbol{R}$, the operation $N_{\boldsymbol{R}}: \mathcal{P}(L \oplus L) \longrightarrow$ $\mathcal{P}(L \oplus L)$ is finitary biconsequence operation.

\footnotetext{
${ }^{1} \mathrm{pr}_{1}$ and $\mathrm{pr}_{2}$ will stand for the first and the second projection respectively, i.e., $\operatorname{pr}_{1}(\langle x, y\rangle):=x$ and $\operatorname{pr}_{2}(\langle x, y\rangle):=y$.
} 
Proof. Because the first two conditions from Definition 2.1 of biconsequence and finiteness are obvious, we will show only that for every set $\boldsymbol{X} \in \mathcal{P}(L \oplus L): N_{\boldsymbol{R}}\left(N_{\boldsymbol{R}}(\boldsymbol{X})_{(1)} \oplus \boldsymbol{X}_{(2)}\right) \subseteq N_{\boldsymbol{R}}(\boldsymbol{X})$.

Assume that $\left(a_{1}, \ldots, a_{n}\right)$ is some biproof from $N_{\boldsymbol{R}}(\boldsymbol{X})_{(1)} \oplus \boldsymbol{X}_{(2)}$ by the rules $\boldsymbol{R}$. We show by induction, that for every $1 \leqslant j \leqslant n$ there exits a biproof $\left(c_{1}, \ldots, c_{m}, a_{j}\right)$ from the set $\boldsymbol{X}$ by using the same set of rules and $\left\{a_{1}, \ldots, a_{n-1}\right\} \subseteq\left\{c_{1}, \ldots, c_{m}\right\}$.

Assume that $j=1$. When $\operatorname{pr}_{1}\left(a_{1}\right) \in N_{\boldsymbol{R}}(\boldsymbol{X})_{(1)}$ and $\operatorname{pr}_{2}\left(a_{1}\right)=1$, then there exists a biproof $\left(c_{1}, \ldots, c_{m}, a_{1}\right)$ from $\boldsymbol{X}$ and our statement holds.

In the case when $\operatorname{pr}_{1}\left(a_{1}\right) \in \boldsymbol{X}_{(2)}$ and $\operatorname{pr}_{2}\left(a_{1}\right)=*: 1$ or $\left(a_{1}\right) \in \bigcup \boldsymbol{R}$, $\left(a_{1}\right)$ is a required biproof.

Assume that the proposition holds for every $i \leqslant j$. If $\left(a_{1}, \ldots, a_{j+1}\right)$ is a biproof and $\left(b_{1}, \ldots, b_{k}, a_{j+1}\right) \in \bigcup \boldsymbol{R}$, for some $\left\{b_{1}, \ldots, b_{k}\right\} \subseteq\left\{a_{1}, \ldots, a_{j}\right\}$, then there are biproofs $\left(c_{1}^{i}, \ldots, c_{m_{i}}^{i}, b_{i}\right)$ for every $i \in\{1, \ldots, k\}$ from $\boldsymbol{X}$ and $\left\{a_{1}, \ldots, a_{j}\right\} \subseteq\left\{c_{1}^{i}, \ldots, c_{m_{i}}^{i}\right\}$. Then $\left(c_{1}^{1}, \ldots, c_{m_{1}}^{1}, \ldots, c_{1}^{k}, \ldots, c_{m_{k}}^{k}\right.$, $\left.b_{1}, \ldots, b_{k}, a_{n+1}\right)$ is the sequence which we needed.

Other cases are the same as in the first part of the proof.

For any biinference $\bar{a}=\left(a_{1}, \ldots, a_{n}\right)$ and $k \in\{0,1, \ldots, n\}$ let us put:

$$
\begin{aligned}
A_{1}^{\bar{a}}(k) & :=\left\{\operatorname{pr}_{1}\left(a_{j}\right) \in L: 1 \leqslant j \leqslant k \& \operatorname{pr}_{2}\left(a_{j}\right)=1\right\}, \\
A_{*: 1}^{\bar{a}}(k) & :=\left\{\operatorname{pr}_{1}\left(a_{j}\right) \in L: 1 \leqslant j \leqslant k \& \operatorname{pr}_{2}\left(a_{j}\right)=*: 1\right\}, \\
A_{*: *}^{\bar{a}}(k) & :=\left\{\operatorname{pr}_{1}\left(a_{j}\right) \in L: 1 \leqslant j \leqslant k \& \operatorname{pr}_{2}\left(a_{j}\right)=*: *\right\} .
\end{aligned}
$$

When biinference is fixed we omit upper index $\bar{a}$.

For any biconsequence $N$ we define the set of birules $\boldsymbol{R}(N)$ :

$r \in \boldsymbol{R}(N)$ iff for every $\bar{a}=\left(a_{1}, \ldots, a_{n}\right) \in r$ and every $\boldsymbol{Y} \in \mathcal{P}(L \oplus L)$ the following condition holds:

- if $A_{1}^{\bar{a}}(n-1) \subseteq N(\boldsymbol{Y})_{(1)}, A_{*: *}^{\bar{a}}(n-1) \subseteq N(\boldsymbol{Y})_{(2)}$ and $N\left(\boldsymbol{Y}_{(1)} \oplus\left(\boldsymbol{Y}_{(2)} \cup\right.\right.$ $\left.A_{*: 1}^{\bar{a}}(n-1)\right)=N(\boldsymbol{Y})$, then

(i) if $\operatorname{pr}_{2}\left(a_{n}\right)=1$, then $\operatorname{pr}_{1}\left(a_{n}\right) \in N(\boldsymbol{Y})_{(1)}$;

(ii) if $\operatorname{pr}_{2}\left(a_{n}\right)=*: *$, then $\operatorname{pr}_{1}\left(a_{n}\right) \in N(\boldsymbol{Y})_{(2)}$;

(iii) if $\operatorname{pr}_{2}\left(a_{n}\right)=*: 1$, then $N\left(\boldsymbol{Y}_{(1)} \oplus\left(\boldsymbol{Y}_{(2)}, \operatorname{pr}_{1}\left(a_{n}\right)\right)=N(\boldsymbol{Y})\right.$.

Lemma 2.1. If $\bar{a}=\left(a_{1}, \ldots, a_{n}\right)$ is a biproof from $\boldsymbol{X}$ by $\boldsymbol{R}(N)$, then $A_{1}^{\bar{a}}(n) \subseteq N(\boldsymbol{X})_{(1)}, A_{*: *}^{\bar{a}}(n) \subseteq N(\boldsymbol{X})_{(2)}$ and $N\left(\boldsymbol{X}_{(1)} \oplus\left(\boldsymbol{X}_{(2)} \cup A_{*: 1}^{\bar{a}}(n)\right)\right)=$ $N(\boldsymbol{X})$. 
Proof. We shall show by induction, that for every $i \in\{1, \ldots, n\}$ :

$\left(*_{i}\right) \quad A_{1}^{\bar{a}}(i) \subseteq N(\boldsymbol{X})_{(1)}, A_{*: *}^{\bar{a}}(i) \subseteq N(\boldsymbol{X})_{(2)}$ and $N\left(\boldsymbol{X}_{(1)} \oplus\left(\boldsymbol{X}_{(2)} \cup A_{*: 1}^{\bar{a}}(i)\right)\right)=N(\boldsymbol{X})$.

It is straightforward to check that $\left(*_{1}\right)$ holds.

Assume that for every $k \leqslant i,\left(*_{k}\right)$ holds, or equivalently $\left(*_{i}\right)$. Let $a_{i+1}$ be introduced to the sequence $\bar{a}$ by $\bar{b}=\left(b_{1}, \ldots, b_{m}, a_{i+1}\right) \in \bigcup \boldsymbol{R}$. Then by the definition of $\boldsymbol{R}(N)$ we have for every $\boldsymbol{Y} \in \mathcal{P}(L \oplus L)$ :

- if $A_{1}^{\bar{b}}(m) \subseteq N(\boldsymbol{Y})_{(1)}, A_{*: *}^{\bar{b}}(m) \subseteq N(\boldsymbol{Y})_{(2)}$ and $N\left(\boldsymbol{Y}_{(1)} \oplus\left(\boldsymbol{Y}_{(2)} \cup\right.\right.$ $\left.A_{*: 1}^{\bar{b}}(m)\right)=N(\boldsymbol{Y})$ then

(i) if $\operatorname{pr}_{2}\left(a_{n}\right)=1$, then $\operatorname{pr}_{1}\left(a_{i+1}\right) \in N(\boldsymbol{Y})_{(1)}$;

(ii) if $\operatorname{pr}_{2}\left(a_{n}\right)=*: *$, then $\operatorname{pr}_{1}\left(a_{i+1}\right) \in N(\boldsymbol{Y})_{(2)}$;

(iii) if $\operatorname{pr}_{2}\left(a_{n}\right)=*: 1$, then $N\left(\boldsymbol{Y}_{(1)} \oplus\left(\boldsymbol{Y}_{(2)}, \operatorname{pr}_{1}\left(a_{i+1}\right)\right)=N(\boldsymbol{Y})\right.$.

In particular for $\boldsymbol{Y}=\left(\boldsymbol{X}_{(1)} \cup A_{1}^{\bar{a}}(i)\right) \oplus\left(\boldsymbol{X}_{(2)} \cup A_{*: 1}^{\bar{a}}(i)\right)$ :

- if $A_{1}^{\bar{b}}(m) \subseteq N\left[\left(\boldsymbol{X}_{(1)} \cup A_{1}^{\bar{a}}(i)\right) \oplus\left(\boldsymbol{X}_{(2)} \cup A_{*: 1}^{\bar{a}}(i)\right)\right]_{(1)}=N(\boldsymbol{X})_{(1)}$, $A_{*: *}^{\bar{b}}(m) \subseteq N\left[\left(\boldsymbol{X}_{(1)} \cup A_{1}^{\bar{a}}(i)\right) \oplus\left(\boldsymbol{X}_{(2)} \cup A_{*: 1}^{\bar{a}}(i)\right)\right]_{(2)}=N(\boldsymbol{X})_{(2)}$ and $N\left[\left(\boldsymbol{X}_{(1)} \cup A_{1}^{\bar{a}}(i)\right) \oplus\left(\left(\boldsymbol{X}_{(2)} \cup A_{*: 1}^{\bar{a}}(i)\right) \cup A_{*: 1}^{\bar{b}}(m)\right)\right]=N\left[\left(\boldsymbol{X}_{(1)} \cup A_{1}^{\bar{a}}(i)\right) \oplus\right.$ $\left.\left(\boldsymbol{X}_{(2)} \cup A_{*: 1}^{\bar{a}}(i)\right)\right]=N(\boldsymbol{X})$, then

(i) if $\operatorname{pr}_{2}\left(a_{i+1}\right)=1$, then $\operatorname{pr}_{1}\left(a_{i+1}\right) \in N\left[\left(\left(\boldsymbol{X}_{(1)} \cup A_{1}^{\bar{a}}(i)\right) \oplus\left(\boldsymbol{X}_{(2)} \cup\right.\right.\right.$ $\left.\left.\left.A_{*: 1}^{\bar{a}}(i)\right)\right)\right]_{(1)} ;$

(ii) if $\operatorname{pr}_{2}\left(a_{i+1}\right)=*: *$, then $\operatorname{pr}_{1}\left(a_{i+1}\right) \in N\left[\left(\left(\boldsymbol{X}_{(1)} \cup A_{1}^{\bar{a}}(i)\right) \oplus\left(\boldsymbol{X}_{(2)} \cup\right.\right.\right.$ $\left.\left.\left.A_{*: 1}^{\bar{a}}(i)\right)\right)\right]_{(2)}$;

(iii) if $\operatorname{pr}_{2}\left(a_{i+1}\right)=*: 1$, then $N\left[\left(\boldsymbol{X}_{(1)} \cup A_{1}^{\bar{a}}(i)\right) \oplus\left(\boldsymbol{X}_{(2)} \cup A_{*: 1}^{\bar{a}}(i)\right.\right.$, $\left.\left.\operatorname{pr}_{1}\left(a_{i+1}\right)\right)\right]=N\left[\left(\boldsymbol{X}_{(1)} \cup A_{1}^{\bar{a}}(i)\right) \oplus\left(\boldsymbol{X}_{(2)} \cup A_{*: 1}^{\bar{a}}(i)\right)\right]$.

But antecedent of this implication is true, thus consequent too, so

- if $\operatorname{pr}_{2}\left(a_{i+1}\right)=1$, then $A_{1}^{\bar{a}}(i+1)=A_{1}^{\bar{a}}(i) \cup\left\{p r_{1}\left(a_{i+1}\right)\right\} \subseteq N\left[\left(\boldsymbol{X}_{(1)} \cup\right.\right.$ $\left.\left.A_{1}^{\bar{a}}(i)\right) \oplus\left(\boldsymbol{X}_{(2)} \cup A_{*: 1}^{\bar{a}}(i)\right)\right]_{(1)}=N(\boldsymbol{X})_{(1)}$;

- if $\operatorname{pr}_{2}\left(a_{i+1}\right)=*: *$, then $A_{*: *}^{\bar{a}}(i+1)=A_{*: *}^{\bar{a}}(i) \cup\left\{\operatorname{pr}_{1}\left(a_{i+1}\right)\right\} \subseteq N\left[\left(\boldsymbol{X}_{(1)} \cup\right.\right.$ $\left.\left.A_{1}^{\bar{a}}(i)\right) \oplus\left(\boldsymbol{X}_{(2)} \cup A_{*: 1}^{\bar{a}}(i)\right)\right]_{(2)}=N(\boldsymbol{X})_{(2)} ;$

- if $\operatorname{pr}_{2}\left(a_{i+1}\right)=*: 1$, then $N\left[\left(\boldsymbol{X}_{(1)} \cup A_{1}^{\bar{a}}(i)\right) \oplus\left(\left(\boldsymbol{X}_{(2)} \cup A_{*: 1}^{\bar{a}}(i)\right), \operatorname{pr}_{1}\left(a_{i+1}\right)\right)\right]$ $=N\left[\left(\boldsymbol{X}_{(1)} \cup A_{1}^{\bar{a}}(i)\right) \oplus\left(\boldsymbol{X}_{(2)} \cup A_{*: 1}^{\bar{a}}(i)\right)\right]=N(\boldsymbol{X})$. 
Cases when $\operatorname{pr}_{1}\left(a_{i+1}\right) \in \boldsymbol{X}_{(1)}, \operatorname{pr}_{2}\left(a_{i+1}\right)=1$ or $\operatorname{pr}_{1}\left(a_{i+1}\right) \in \boldsymbol{X}_{(2)}$, $\operatorname{pr}_{2}\left(a_{i+1}\right)=*: 1$ are straightforward. Finally $\left(*_{i+1}\right)$ holds.

Theorem 2.3. For every $\boldsymbol{X} \in \mathcal{P}(L \oplus L): N_{\boldsymbol{R}(N)}(\boldsymbol{X}) \subseteq N(\boldsymbol{X})$. Moreover, if $N$ is finitary, then $N_{\boldsymbol{R}(N)}=N$.

Proof. When $\langle\alpha, x\rangle \in N_{\boldsymbol{R}(N)}(\boldsymbol{X})$, then there exists a biproof $(\bar{a},\langle\alpha, x\rangle)$ from the set $\boldsymbol{X}$ by using the rules $\boldsymbol{R}(N)$. According to Lemma 2.1, $\langle\alpha, x\rangle \in N(\boldsymbol{X})_{(1)}$, when $x=1 ;\langle\alpha, x\rangle \in N(\boldsymbol{X})_{(2)}$, when $x=*: *$. Moreover, $\langle\alpha, x\rangle \in N\left(\boldsymbol{X}_{(1)} \oplus\left(\boldsymbol{X}_{(2)}, \alpha\right)\right)$, and consequently $\alpha \in N\left(\boldsymbol{X}_{(2)}\right.$, when $x=*: 1$.

If $\alpha \in N(\boldsymbol{X})_{(1)}$, then $\alpha \in N\left(\left\{\beta_{1}, \ldots, \beta_{n}\right\} \oplus\left\{\gamma_{1}, \ldots, \gamma_{k}\right\}\right)_{(1)}$, for some $\beta_{1}, \ldots, \beta_{n} \in \boldsymbol{X}_{(1)}$ and $\gamma_{1}, \ldots, \gamma_{k} \in \boldsymbol{X}_{(2)}$. Then $\bar{a}=\left(\left\langle\beta_{i}, 1\right\rangle_{i=1}^{n},\left\langle\gamma_{i}\right.\right.$, $\left.*: 1\rangle_{i=1}^{k}\langle\alpha, 1\rangle\right)$ is the biinference which fulfils both $\bar{a} \in \bigcup \boldsymbol{R}(N)$ and $\bar{a}$ is a biproof from $\boldsymbol{X}$ using $\boldsymbol{R}(N)$. Similarly in the case when $\alpha \in N(\boldsymbol{X})_{(2)}$ (it is enough biproof terminating on $\langle\alpha, *: *\rangle$ ).

Definition 2.5. For any class of similar bimartix $B M=\left\{\left\langle M^{t}, F_{1}^{t}, \ldots\right.\right.$, $\left.\left.F_{n}^{t}, D_{1}^{t}, D_{*: 1}^{t}, D_{*: *}^{t}\right\rangle: t \in T\right\}$, we shall say that birule is valid for $B M$ (or BM-valid) iff for each $\bar{a}=\left(a_{1}, \ldots, a_{k}\right) \in r, t \in T$ and $h_{t} \in \operatorname{Hom}\left(\mathfrak{L},\left\langle M^{t}\right.\right.$, $\left.\left.F_{1}^{t}, \ldots, F_{n}^{t}\right\rangle\right): h_{t}\left(A_{x}^{\bar{a}}(k-1)\right) \subseteq D_{x}^{t}$ for $x=1, *: 1, *: * \operatorname{implies} \operatorname{pr}_{1}\left(a_{k}\right) \in$ $D_{\mathrm{pr}_{2}\left(a_{k}\right)}$. We put $\boldsymbol{R}(\boldsymbol{B M})$ for the set of $\boldsymbol{B} \boldsymbol{M}$-valid birules.

Theorem 2.4. For any class of bimatrices $B M$ and any $\boldsymbol{X} \in \mathcal{P}(L \oplus L)$ : $N_{\boldsymbol{R}(B M)}(\boldsymbol{X}) \subseteq N_{B M}(\boldsymbol{X})$. Moreover, if $N_{B M}$ is finitary, then $N_{\boldsymbol{R}(\boldsymbol{B M})}=$ $N_{B M}$.

Proof. For the first part it is enough to show by induction that for any biproof $\bar{a}=\left(a_{1}, \ldots, a_{k}\right)$ from the set $\boldsymbol{X} \in \mathcal{P}(L \oplus L)$ and any $h_{t}$ from $\operatorname{Hom}\left(\mathfrak{L},\left\langle M^{t}, F_{1}^{t}, \ldots, F_{n}^{t}\right\rangle\right)$ :

if $h_{t}\left(A_{x}^{\bar{a}}(k-1)\right) \subseteq D_{x}^{t}$ for $x=1, *: 1, *: *$, then $\operatorname{pr}_{1}\left(a_{k}\right) \in D_{\operatorname{pr}_{2}\left(a_{k}\right)}$.

The proof of this fact is easy but quite long. Moreover it is very similar to the case of $p$-consequence (see [2]).

Assume that $N_{B M}$ is finitary and $\alpha \in N_{B M}(\boldsymbol{X})_{(i)}$, i.e. $\alpha \in N_{B M}\left(\boldsymbol{X}_{f}\right)_{(i)}$ for some $\boldsymbol{X}_{f}=\left\{\left\langle\chi_{j}, x_{j}\right\rangle\right\}_{j=1}^{k}$ and finite subset of $\boldsymbol{X}\left(x_{j} \in\{1,2\}\right)$. It is easy to check, that $\left(\left\langle\chi_{j}, f x_{j}\right\rangle_{i=1}^{k},\langle\alpha, 1\rangle\right)$, when $i=1$, and $\left(\left\langle\chi_{j}, f x_{j}\right\rangle_{i=1}^{k}\right.$, $\langle\alpha, *: 1\rangle)$, when $i=2$, is desirable biproof. We have used notation $f:\{1,2\} \longrightarrow\{1, *: 1\}, f(1)=1$ and $f(2)=*: 1$. 


\section{Operations associated with biconsequence}

For any biconsequence $N$ and arbitrary but fixed $Y \subseteq L$ we define $C_{Y}^{N}: \mathcal{P}(L) \rightarrow \mathcal{P}(L)$, by putting for any $X \in \mathcal{P}(L)$ :

$$
C_{Y}^{N}(X):=N(X \oplus Y)_{(1)} .
$$

We note without proofs.

FACT 3.1. $C_{Y}^{N}$ is consequence operation. If $N$ is finitary (resp. structural), then so is $C_{Y}^{N}$.

For any consequences $C_{1}, C_{2}$ for the same language by $C_{1} \leq C_{2}$ we note the fact, that for every $X \in \mathcal{P}(L): C_{1}(X) \subseteq C_{2}(X)$. Naturally $\leq$ is a partial order.

FACT 3.2. $C_{Y_{1}}^{N} \leq C_{Y_{2}}^{N}$ whenever $Y_{1} \subseteq Y_{2}$.

Implication in the opposite direction does not hold. For example we can define

$$
N(X \oplus Y):= \begin{cases}L \oplus L & \text { if } Y \neq \emptyset \\ X \oplus \emptyset & \text { if } Y=\emptyset\end{cases}
$$

then for all non-empty sets $Y_{1}$ and $Y_{2}$ we have that $C_{Y_{1}}^{N}=C_{Y_{2}}^{N}$.

For arbitrary family of consequences $C$ we put $\bigvee C$ for supremum of $C$ in the lattice of consequences for the language $\mathcal{L}$.

FACT 3.3. If $N$ is finitary and $\left\{Y_{\zeta}\right\}_{\zeta<\xi}$ is a chain of sets of formulas (where $\xi$ is an ordinal), then

$$
\bigvee_{\zeta<\xi} C_{Y_{\zeta}}^{N}=C_{\bigcup_{\zeta<\xi}^{N} Y_{\zeta}} .
$$

FACT 3.4. If $N$ is finitary and $\left\{Y_{t}\right\}_{t \in T}$ is a directed set of sets of formulas, then

$$
\bigvee_{t \in T} C_{Y_{t}}^{N}=C_{\bigcup_{t \in T}^{N} Y_{t}} .
$$




\section{Final remarks and conclusions}

So far it was proved a few properties of biconsequence operation. The higher complexity of this notion allows for considering many classes of biconsequences. In the fact, we have consider the most general case of biconsequence, but it is possible to take under considerations such operations $N$ for which the following condition is valid:

$$
N(\boldsymbol{X})_{(1)} \subseteq N(\boldsymbol{X})_{(2)} .
$$

It can be interpreted as the fact that every "good" conclusion is a "worse" consequence. For example the sentence ' $2+2=4$ ' is universally valid (in the standard meaning of symbols), so it is a conclusion of the right side as well (likewise in the theory of $p$-consequence).

But there arises another problem. We have three types of sentences:

- sentences that occur on the left side (good justified in the new sensedistinguished by the symbol 1) and,

- sentences from the right side: worse-good, and worse-worse (associated to $*: 1$ and $*: *$, respectively).

It would seems that our problem has been moved but not removed, namely, how to distinguish worse-good from worse-worse sentences. Our explanation is rather simple - we have the most fundamental distinction between good justified and not-rejected sentences and the rest is out of our interest. For example, the first component of a disjoint sum can correspond to undoubtedly true sentences (of the mathematical character), the other one can contain statements concerning the material world. Both types of sentences could be naturally expressed in some language containing both of them.

One more thing - it is possible to multiple a number of components in disjoint sum. Every of them would be to correspond of different degree of sureness (we remark that degrees not need to be linear a order). But it is something which requires more reflection, and we leave it for a future work.

\section{References}

[1] Ajdukiewicz, K., Pragmatic Logic, Dordrecht, Reidel, 1974.

[2] Frankowski, Sz., "Formalization of plausible inference", Bulletin of the Section of Logic 33, 1 (2004): 41-52. 
[3] Frankowski, Sz., " $p$-consequence versus $q$-consequence operations", Bulletin of the Section of Logic 33, 4 (2004): 195-207.

[4] Frankowski, Sz., "Plausible reasoning expressed by $p$-consequence", Bulletin of the Section of Logic 37, 3-4 (2008): 161-170.

[5] Frankowski, Sz., "On the lattice of p-consequences, Reports on Mathematical Logic 45 (2010): 23-35.

[6] Shramko, Y., and H. Wansing, "Entailment relations and/as truth values", Bulletin of the Section of Logic 36, 3-4 (2007): 131-144.

SZYMON FRANKOWSKI

Department of Logic

University of Łódź

Łódź, Poland

frankowski@filozof .uni.lodz.pl 